\title{
Tactile change detection
}

\author{
Alberto Gallace ${ }^{*} \quad$ Hong Z. Tan ${ }^{\dagger} \quad$ Charles Spence ${ }^{*}$ \\ (')Department of Experimental Psychology, Oxford University, Oxford, UK \\ (ł)Dipartimento di Psicologia, Universita' degli Studi di Milano Bicocca, Milano, Italy \\ (†)Haptic Interface Research Laboratory, Purdue University, West Lafayette, USA \\ E-mail: alberto.gallace@psy.ox.ac.uk, hongtan@purdue.edu, charles.spence@psy.ox.ac.uk
}

\begin{abstract}
Interest in the use of tactile information displays has grown rapidly in recent years. However, relatively little research has been conducted to explore any cognitive and/or attentional limitations that may be inherent when using the body as a receptor surface for the transmission of information. In the present study, participants attempted to detect changes to tactile patterns presented sequentially on the body surface. The patterns consisted of 1-3 vibrotactile stimuli presented for 200ms with a blank interstimulus interval of $800 \mathrm{~ms}$. The pattern of tactile stimulation was repeatedly changed (alternating between two different patterns) on 50\% of the trials, while no change occurred on the remaining trials. The results showed that participants often failed to detect the changes to the consecutively-presented tactile patterns. This finding may reflect a tactile equivalent of the phenomenon of change blindness reported in previous visual studies. The implications of these finding for human tactile interface design are discussed.
\end{abstract}

\section{Introduction}

The last few years has seen a rapid growth of interest in the development and utilization of tactile interfaces [1-5]. In part, this interest reflects the growing belief amongst many applied researchers that the visual and auditory modalities may have become overloaded in many contemporary interface environments [6,7]. The hope is that the tactile modality might therefore offer an important (but at present relatively underutilized) means of presenting information to interface operators in situations where the other sensory modalities (for whatever reason) cannot be used, or else are already overloaded [3].

Recent research has demonstrated that tactile displays can be used to convey information under conditions of high gravitational load when visual information is severely degraded [7]. Studies have also shown that tactile stimulators on the body surface can be used to successfully resolve spatial disorientation in pilots [3], as well to convey aircraft position and motion information [8]. Tactile interfaces have also been used to support orientation awareness for astronauts in micro-gravity environments [9], and to cue driver attention in cars $[1,7]$.

Although the body surface clearly offers a potentially important alternative means of conveying information to an interface operator, several fundamental questions concerning the limitations and/or advantages of utilizing tactile information displays have yet to be addressed empirically [6,10]. These include questions related to the kinds of information that are best conveyed by touch, how many tactile sources of information can be perceived at any one time $[11,12]$, and how well people can detect changes to ongoing patterns of tactile stimulation [1315]. In the present study, we explored the ability of normal adult human observers to perceive changes to simple sequentially-presented tactile patterns consisting of between 1-3 vibrotactile stimuli distributed over the body surface.

\section{Methods}

\subsection{Participants}

13 right-handed participants $(6$ males and 7 females) took part in this experiment as paid volunteers (mean age of 27.3 years, range of 19-32 years). All of the participants reported normal tactile perception. The experiment was non-invasive and had ethical approval from the Department of Experimental Psychology, Oxford University. The experiment was performed in accordance with the ethical standards laid down in the 1964 Declaration of Helsinki. 


\subsection{Apparatus and materials}

The experiment was conducted in a normally illuminated room. The participants sat comfortably on a chair for the duration of the study. The vibrotactile stimuli were presented by means of seven resonanttype tactors (Part No: VBW32, Audiological Engineering Corp., Somerville, MA, USA), with $1.6 \mathrm{x}$ $2.4 \mathrm{~cm}$ vibrating surfaces. The tactors were placed on the participant's body over the top of their clothing by means of Velcro strip belts (see Figure 1 for the position of the tactors on the body). The participants were not able to see any of the tactors directly underneath the belts. We selected the 7 body sites where stimulation might be applied on the basis of their relative "salience" in order to minimize localization errors [cf. 11,12]. The vibrators were driven by means of a custom-built 9-channel amplifier circuit that drove each tactor independently at $290 \mathrm{~Hz}$ (close to its resonant frequency). The intensity and the on/off timing of each tactor was controlled through the serial port of a laptop computer running custom software written in Matlab 6.0. White noise was presented over closed ear headphones at $70 \mathrm{~dB}(\mathrm{~A})$ to mask any sounds made by the operation of the vibrotactile stimulators.

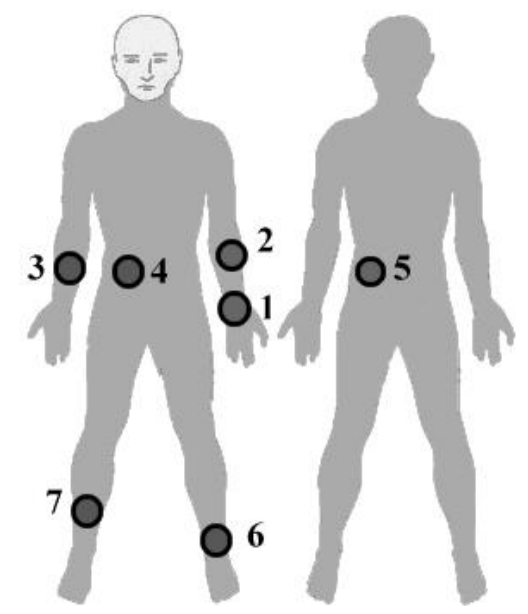

FIG. 1. Positions on the body surface where the tactors were placed: 1) left wrist; 2) just below the left elbow; 3) midway between the wrist and elbow on the right arm; 4) on the waistline, to the right of the body midline; 5 ) on the back, to the left of the body midline; 6) just above the left ankle; and 7) midway between the ankle and knee on the right leg. Note that these positions were chosen to ensure that homologous sites on both sides of the body were never stimulated.
The intensity of each tactor was adjusted individually at the beginning of the experiment. The participants were requested to judge whether the intensity of each of the vibrotactile stimuli was high enough to be perceived clearly. The participants were also requested to match the intensities of the seven tactors, so that all of the stimuli were perceived to be of a similar intensity. The participants adjusted the tactor intensity in the same order of tactor 1 to 7 (see Figure 1). The amplification levels for the seven tactors were kept at their individually-chosen levels throughout the experiment.

A)

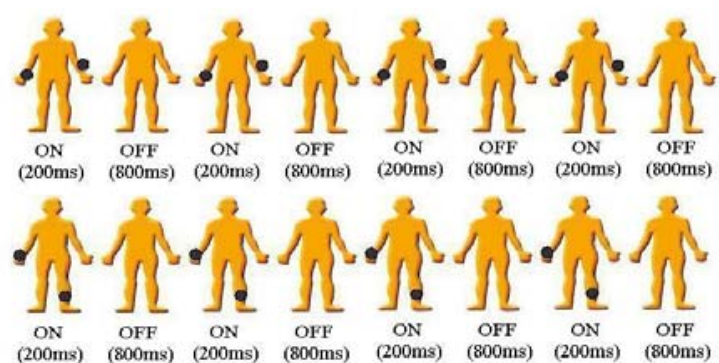

B)
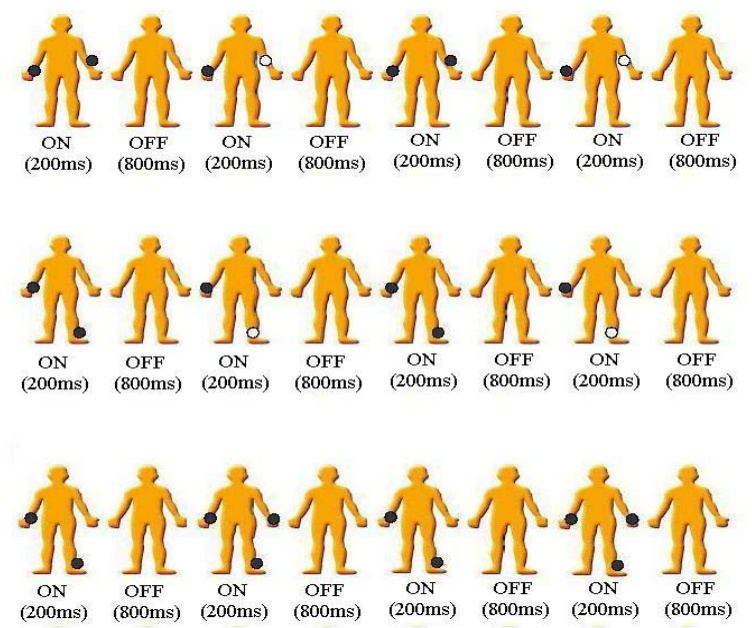

FIG. 2. Examples of some of the vibrotactile stimulus patterns used in the experiment. Each line represents the first 4 seconds of a given trial. The filled black circles represent the activated tactors. A) 2 different No Change conditions. B) 3 different Change conditions. The filled black circles represent the activated tactors and the white circles represent the deactivated tactor in the Offset trials. The first two lines represent Offset trials and the third shows an Onset trial. 
On each trial, the stimuli consisted of two $200 \mathrm{~ms}$ patterns of vibrotactile stimulation. An $800 \mathrm{~ms}$ empty interstimulus interval (ISI) followed the presentation of each pattern. The presentation of the stimuli continued until a response had been made or until 10 seconds had elapsed, at which time the trial was terminated (see Figure 2). The first tactile pattern to be presented on each trial always consisted of 2 tactors activated at a variety of different body sites (note that all of the 128 possible different pairs of body sites were stimulated at some point during the experiment). In the No-change condition, the same vibratory pattern was presented repeatedly throughout the trial. In the Change condition, the second (or alternate) vibratory pattern was either composed of only one of the 2 tactors activated in the first pattern (Offset condition), or of the same vibratory pattern as in the first presentation plus one additional tactor (Onset condition). Note that the number of tactors activated at any one time never exceeded a maximum of 3 . This constraint was incorporated into our experimental design because recent research has shown that the ability of participants to detect simultaneously presented stimuli over the body surface declines quite rapidly as the number of tactors activated increases above this number [11].

\subsection{Procedure}

The participants were instructed to press one of two keys on a computer keyboard as soon as they decided whether or not a change was present in a given trial. The stimuli were presented until a response was made or until the trial was terminated (this occurred if no response had been made within 10 seconds of the onset of the display). In $50 \%$ of trials, the pattern changed (i.e., alternated) between successive stimulus presentations (equiprobably an onset or offset change), while in the remaining trials, no change occurred (i.e., the same display was repeatedly presented throughout the trial). Each participant completed 504 trials in total, in an experimental testing session lasting approximately 45 minutes.

\section{Results}

Trials in which participants failed to make a response before the trial was terminated (less than $1 \%$ of trials overall) were not included in the data analyses. The mean reaction time (RT) and error rates for the Change and No Change conditions were calculated for each participant on the basis of the remaining trials. Paired t-tests revealed a significant difference between the conditions in terms of the error data $[\mathrm{t}(12)=4.71 ; \mathrm{p}<$ $.0005]$, while the trend in the RT data failed to reveal significance $[\mathrm{t}(12)=1.83 ; \mathrm{p}=.09]$. Participants made significantly more errors and responded somewhat more slowly in the Change condition than in the No Change condition $(16.5 \%$ errors and a mean RT of 3840 ms versus $4.7 \%$ errors and a mean RT of 3530 ms, respectively; see Figure 3A).

A)

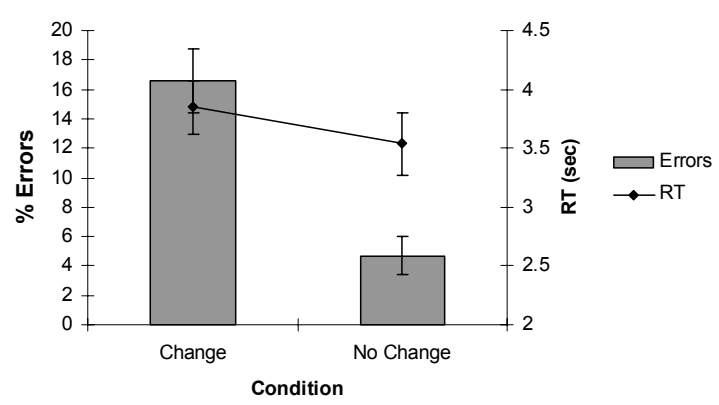

B)

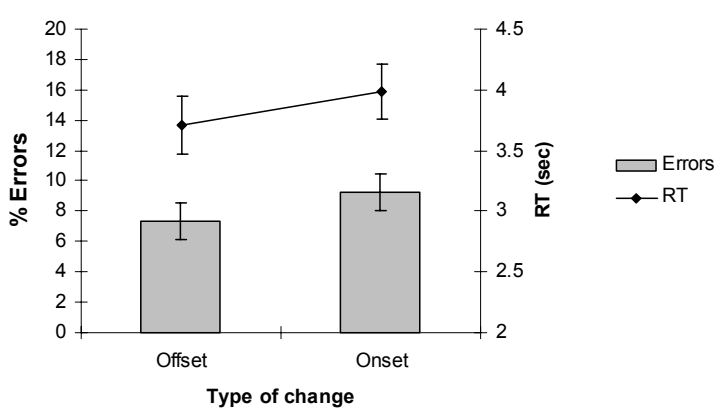

FIG. 3. A) Mean error rates and RTs in the Change and No Change conditions. The scale on the left represents the percentage of errors while the scale on the right represents the mean latency of participant's responses. B) Mean error rates and RTs as in the Offset and Onset trials in the Change condition. The left scale represents the percentage of errors while the right scale represents the mean latency of participant's responses. Error bars represent the standard errors of the means.

Mean RTs and error rates were then calculated separately for the Onset and Offset trials in the Change condition. Paired t-tests revealed a significant difference between the Onset and Offset trials in terms of the RT data $[\mathrm{t}(12)=-2.37 ; \mathrm{p}<.05]$. However, the trend in the error data just failed to reach statistical significance $[\mathrm{t}(12)=-1.96 ; \mathrm{p}=.07]$. Participants responded significantly more slowly, and somewhat 
less accurately, on Onset trials than on Offset trials $(9.2 \%$ and $3980 \mathrm{~ms}$ versus $7.2 \%$ and $3710 \mathrm{~ms}$, respectively see Figure 3B).

\section{Discussion}

The results of the experiment reported here reveal that people are unable to reliably detect when even a simple tactile pattern (consisting of only 1-3 vibrotactile elements over the body surface) changes, providing that the interval between successive pattern presentations is at least $800 \mathrm{~ms}$ long. This finding has important ramifications for the future design and implementation of tactile information displays, especially when it is realized that the perceptual load in any real-life interface environment is likely to be much higher than that under which participants were performing the present study [7], hence leading to the suggestion that change detection performance may be even worse in real life situations. The consequences of the manipulation of perceptual load [16] on tactile pattern perception are now the subject of an ongoing series of experiments in our laboratory.

Over the last fifty, years a large body of research has revealed a surprising inability of people to detect changes that are suddenly introduced into visual scenes [17-19]. This inability to detect change, a phenomenon known as change blindness in vision (see [19], for a review) has also been reported to affect auditory perception, where it is known as "change deafness" $[20,21]$. The results of the present study show for the first time that deficits in change detection can also influence the perception of tactile patterns presented over the body surface.

Our results also show that participants detected changes in Onset trials significantly more accurately than changes in Offset trials. Although a similar pattern of results has been reported previously in studies of visual change blindness [22], it is important to note that the present findings may have more to do with the absolute number of tactile stimuli presented in Onset versus Offset displays rather than the relative detectability of onsets versus onsets per se. Once again, clarification of this issue will have to await further research.

It will also be interesting in future research to find out how performance changes as the ISI between two changing patterns is varied. Our prediction, based on the existing literature on visual change blindness [23], is that change detection performance should improve quite dramatically as the interval between pattern presentation is reduced. Such an improvement may reflect the consequences of any tactile apparent motion cues [24,25] that emerge at shorter ISIs (i.e., in the range of 100-200 ms). Similarly, reducing the ISI should also serve to ensure that both the original and changed tactile displays are presented within the temporal limits of tactile short term memory [26].

It will certainly be an interesting question for future research to address the question of what role attention plays in the perception of change in tactile displays such as those used here. Indeed, research in this area may relate more directly to real world applications in the area of human tactile interface design: For instance, one might expect that in many real-life situations, where attentional resources need to be allocated to a variety of highly-demanding concurrent tasks/stimuli, tactile change detection performance might be substantially worse. Such a result would clearly highlight an important potential limitation inherent in the use of tactile interfaces to accurately convey different kinds of information to interface operators, especially when the nature of that information is likely to change over time.

In conclusion, the results of the present study provide the first empirical demonstration of the existence of a tactile equivalent of the phenomenon of change blindness reported previously in studies of visual scene perception. Specifically, our results show that people frequently fail to notice when a change is introduced into an alternating tactile pattern presented over the body surface, at least at the $800 \mathrm{~ms}$ ISIs used here. This failure to notice tactile change occurred even when change detection was the participant's only task (i.e., when the perceptual load of the experimental situation was relatively low). As such, problems with tactile change detection may therefore be much worse in many real-world environments where interface designers might consider implementing tactile information displays (e.g., see [27]).

\section{References}

[1] C. Ho, H. Z. Tan, \& C. Spence. Using spatial vibrotactile cues to direct a driver's visual attention. Transportation Research Part F, 2004, submitted.

[2] J. L. Rochlis \& D. J. Newman. A tactile display for international space station (ISS) extravehicular activity (EVA). Aviation, Space, and Environmental Medicine 71, 2000, pp. 571-578.

[3] A. H. Rupert. An instrumentation solution for reducing spatial disorientation mishaps. IEEE Engineering in Medicine and Biology 19, 2000, pp. 71-80. 
[4] R. D. Sorkin. Design of auditory and tactile displays. In G. Salvendy (Ed.), Handbook of human factors (pp. 549576), 1987, New York: Wiley.

[5] J. B. F. Van Erp. Tactile navigation display. Haptic Human-Computer Interaction 2000, 2000, pp. 165-173.

[6] C. Spence \& J. Driver. Cross-modal links in attention between audition, vision, and touch: Implications for interface design. International Journal of Cognitive Ergonomics 1, 1997, pp. 351-373.

[7] H. A. H. C. Van Veen \& J. B. F. van Erp. Tactile information presentation in the cockpit. Haptic HumanComputer Interaction 2000, 2000, pp. 174-181.

[8] A. H. Rupert, F. E. Guedry, \& M. F. Reschke. The use of tactile interfaces to convey position and motion perceptions. Advisory Group for Aerospace Research and Development CP541 20 1, 1994, pp. 20-75.

[9] J. B. F. Van Erp, \& H. A. H. C. Van Veen. A multipurpose tactile vest for astronauts in the international space station. In Proceedings of Eurohaptics 2003 (pp. 405-408), 2003, Dublin, Ireland: Trinity College.

[10] J. M. Loomis. Tactile pattern perception. Perception 10, 1979, pp. 5-27.

[11] A. Gallace, H. Z. Tan, \& C. Spence. Numerosity judgments for tactile stimuli distributed over the body surface. Perception, 2004, submitted.

[12] F. A. Geldard \& C. E. Sherrick. Multiple cutaneous stimulation: The discrimination of vibratory patterns. Journal of the Acoustical Society of America 37, 1965, pp. 797-801.

[13] J. C. Bliss, H. D. Crane, S. W. Link, \& J. T. Townsend Tactile perception of sequentially presented spatial patterns. Perception \& Psychophysics 1, 1966, pp. 125-130.

[14] A. P. Hillstrom, K. Shapiro, \& C. Spence. Attentional and perceptual limitations in processing sequentially presented vibrotactile targets. Perception \& Psychophysics 64, 2002, pp. 1068-1082.

[15] S. Soto-Faraco, C. Spence, K. Fairbank, A. Kingstone, A. P. Hillstrom, \& K. Shapiro. A crossmodal attentional blink between vision and touch. Psychonomic Bulletin \& Review 9, 2002, pp. 731-738.

[16] G. Rees \& N. Lavie. What can functional imaging tell us about the role of attentional load in awareness? Neuropsychologia 39, 2001, pp. 1343-1353.

[17] R. S. French. The discrimination of dot patterns as a function of number and average separation of dots. Journal of Experimental Psychology 46, 1953, pp. 1-9.
[18] J. Hochberg. In the mind's eye. In R. N. Haber (Ed.), Contemporary theory and research in visual perception (pp. 309-331), 1968, New York: Holt, Rhinehart \& Winston.

[19] R. A. Rensink. Change detection. Annual Review of Psychology 53, 2002, pp. 245-277.

[20] J. S. Chan \& C. Spence. Change deafness: An auditory analogue of visual change blindness? Perception, 2004, submitted.

[21] M. S. Vitevitch. Change deafness: The inability to detect changes between two voices. Journal of Experimental Psychology: Human Perception and Performance 29, 2003, pp. 33-342.

[22] G. G. Cole, R. W. Kentridge, A. R. H. Gellatly, \& C. A. Heywood. Detectability of onsets versus offsets in the change detection paradigm. Journal of Vision 3, 2003, pp. 22-31.

[23] D. J. Simons \& D. T. Levin. Change blindness. Trends in Cognitive Sciences 1, 1997, pp. 261-267.

[24] J. H. Kirman. Tactile apparent movement: The effects of interstimulus onset interval and stimulus duration. Perception \& Psychophysics 15, 1974, pp. 1-6.

[25] S. Lakatos \& R. N. Shepard. Constraints common to apparent motion in visual, tactile, and auditory space. Journal of Experimental Psychology: Human Perception \& Performance 23, 1997, pp. 1050-1060.

[26] C. Miles \& M. Borthwick. Tactile short-term memory revisited. Memory 4, 1996, pp. 655-668.

[27] J. B. F. Van Erp \& H. A. H. C. Van Veen. Vibrotactile in-vehicle navigation system. Transportation Research Part F 7, 2004, pp. 247-256.

\section{Acknowledgments}

A. G. was supported by a grant from the Universita' di Milano Bicocca, Italy. H. Z. T. and C. S. were supported by a Network Grant from the Oxford McDonnell-Pew Centre for Cognitive Neuroscience. Correspondence regarding this article should be addressed to Alberto Gallace, Room B121, Department of Experimental Psychology, University of Oxford, Oxford, UK, OX16UD.

E-mail: alberto.gallace@psy.ox.ac.uk. 